\title{
ON THE SHEAF $H_{n}$ OF HIGHER HOMOTOPY GROUPS AS AN ABELIAN COVERING SPACE
}

S. BALCI and E. GÜNER

Department of Mathematics, University of Ankara, Ankara, TURKEY

(Received Oct. 31, 1997; Revised March 20, 1998; Accepted April 3, 1998)

\begin{abstract}
Let $\mathrm{X}$ be a connected and locally path connected topological space. Constructing the sheaf $\mathrm{H}$ of higher homotopy groups on $\mathrm{X}$, its some characterizations are examined. Also, it is shown that $\mathbf{H}$ is a regular covering space as a sheaf of abelian groups. Finally, it is given "General Lifting Theorem" for the sheaf $H$ and constructing the Quotient Sheaf $Q_{H \text { " }}$ for any group subsheaf $\mathrm{H}^{\prime}$ of the sheaf $\mathrm{H}_{\mathrm{n}}$, it is shown that $\mathrm{Q}_{\mathrm{H}_{\mathrm{n}}}$ is a covering space as sheaf of abelian groups.
\end{abstract}

\section{INTRODUCTION}

Let $X$ be a connected and locally path connected topological space. Then, $X$ is a path connected. For an arbitrary fixed point $c \in X$, we will consider $X$ as a pointed topological space $(X, c)$ unless otherwise stated. Let $X$ be any point of $X$ and $\pi_{n}(X, x)$ be higher homotopy groups of $X$ with respect to $x$ and

$$
H_{n}=\underset{x \in X}{V} \pi_{n}(X, x) \text {. }
$$

Clearly, $H_{n}$ is a set over $X$ and the mapping $\Psi: H_{n} \rightarrow X$ defined by $\Psi$ $\left(\sigma_{x}\right)=x$ for any $\sigma_{x} \in\left(H_{n}\right)_{x} \subset H_{n}$, is an onto projection.

We introduce on $H_{n}$ a natural topology as follows: Let $x_{0}$ an arbitrary fixed point of $\mathrm{X}, \mathrm{W}=\mathrm{W}\left(\mathrm{x}_{0}\right)$ be a path connected open neighborhood of $x_{0}$ and $\sigma_{x_{0}}=[\alpha]_{x_{0}}$ be a homotopy class of $\left(H_{n}\right)_{x_{0}}$. Since $X$ path connected, there exists a path $\gamma$ with initial point $x_{0}$ and with terminal point $x$, for every $x \in W$. Therefore, the path $\gamma$ determines an isomorphism $\gamma^{*}:\left(\mathrm{H}_{\mathrm{n}_{\mathrm{x}_{0}}} \rightarrow\left(\mathrm{H}_{\mathrm{n}{ }_{\mathrm{x}}}\right.\right.$ defined by $\gamma^{*}\left([\alpha]_{\mathrm{x}_{0}}\right)=[\beta]_{\mathrm{x}}$ for any $[\alpha]_{\mathrm{x}_{0}}$ $\in\left(\mathrm{H}_{\mathrm{n} \mathrm{x}_{0}} \subset \mathrm{H}_{\mathrm{n}}\right.$. Let us now define a mapping $\mathrm{s}: \mathrm{W} \rightarrow \mathrm{H}_{\mathrm{n}}$ such that 
$s(x)=\gamma^{*}\left([\alpha]_{x_{0}}\right)=[\beta]_{x}$ for every $x \in W$. If $c \in W$, then we define $s(c)=$ $\gamma^{*}\left([\alpha]_{c}\right)=[\alpha]_{c}$, by taking $[\gamma]=[1] \in\left(H_{n}\right)_{c}$. It is seen that, the mapping $s$ depends on both the homotopy classes $[\alpha]_{x_{0}}$ and $[\gamma]$. Suppose that the homotopy class $[\gamma]$ is choosen as arbitrary fixed, for each $\mathrm{x} \in \mathrm{W}$. So, the mapping $s$ depends on only the homotopy class $[\alpha]_{x_{0}} \cdot s$ is well-defined and $\Psi o s=1_{w}$. Let us denote the totality of the mapping $s$ defined over $W$ by $\Gamma\left(W, H_{n}\right)$.

Let $\mathrm{B}$ a basis of path conneted open neighborhoods for each $\mathrm{x} \in \mathrm{X}$. Then,

$$
\mathrm{T}_{\mathrm{n}}=\left\{\mathrm{s}(\mathrm{W}): \mathrm{W} \in \mathrm{B}, \mathrm{s} \in \Gamma\left(\mathrm{W}, \mathrm{H}_{\mathrm{n}}\right)\right\}
$$

is a topology - base on $\mathrm{H}_{n}[4,10]$. In this topology the mapping $\Psi$ and $\mathrm{s}$ are continuous. Moreover $\Psi$ is a local topological mapping and the mapping $s$ is a local invers of $\Psi$. Because;

1. Let $\sigma_{x_{0}} \in H_{n}$. Then $\Psi\left(\sigma_{x_{0}}\right)=x_{0} \in X$. If $W=W\left(x_{0}\right)$ is an open set, then $W=U_{i \in 1} W_{i}$, where each $W_{i} \in B$. So, for each $W_{i}$, there exists a mapping $s_{i}: W_{i} \rightarrow H_{n}$ such that $\Psi_{0} s_{i}=1_{w_{i}}$ and $s_{i}\left(W_{i}\right) \in T_{n}$.

Let us now define a mapping $s: W \rightarrow H_{n}$ such that $s / W_{i}=s_{i}$, for each $W_{i}$. Thus

$$
s(W)=\bigcup_{i \in I} s_{i}\left(W_{i}\right)
$$

is an open set in $H_{n}$ and $\Psi o s=1_{w}$. Write $s(W)=U$. Since $\Psi o s=1_{w}$, so $\Psi=1_{U}$, then $\Psi \mid U: U \rightarrow W$ is bijective and $(\Psi \mid U)^{-1}=s$.

2. The topologies on $U$ and $W$ are subspace topologies obtained from $H_{n}$ and $X$, respectively. Let $W^{\prime} \subset W$ be an open set. It can be written that

$$
W^{\prime}=\bigcup_{i \in I} W_{i}^{\prime}
$$

such that $W_{i}^{\prime}=W_{i}^{\prime} \cap W^{\prime}$ for any $i \in I$. Now, if we define a mapping

$$
\mathrm{s}_{\mathrm{i}}^{\prime}: \mathrm{W}_{\mathrm{i}}^{\prime} \rightarrow \mathrm{U}
$$

such that $s_{i}^{\prime}=s_{i} \mid W_{i}^{\prime}$, for each $W_{i}^{\prime}$, then we can define a mapping 


$$
\mathrm{s}^{\prime}: \mathrm{W}^{\prime} \rightarrow \mathrm{U}
$$

such that $s^{\prime} \mid W_{i}^{\prime}=s_{i}^{\prime}$. So,

$$
s^{\prime}\left(W^{\prime}\right)=\bigcup_{i \in I} s_{i}^{\prime}\left(W_{i}^{\prime}\right) \subset U
$$

is an open set. Hence $\Psi / U$ is a continuous mapping. On the other hand, if $U^{\prime} \subset U$ is an open set, then

$$
U^{\prime}=U_{i \in I} s_{i}^{\prime}\left(W_{i}^{\prime}\right)
$$

Hence

$$
\Psi\left(\mathrm{U}^{\prime}\right)=\mathrm{U}_{\mathrm{i} \in \mathrm{l}} \mathrm{W}_{\mathrm{i}}^{\prime} \subset \mathrm{W}
$$

is an open set. Thus, the mapping $\mathrm{s}: \mathrm{W} \rightarrow \mathrm{U}$ is continuous.

Therefore $\left(H_{n}, \Psi\right)$ is a sheaf over $X$. It is called "the sheaf of higher homotopy groups" [6]. $\mathrm{s}$ is called a section over $\mathrm{W}$ and the set of totality of sections over $W$ is $\Gamma\left(W, H_{n}\right)$. The $\left(H_{n}\right)_{x}=\pi_{n}(X, x)$ is called the stalk of the sheaf $H_{n}$ for any $x \in X$. The group $\left(H_{n}\right)_{x}{ }^{n}=\pi_{n}(X, x), n>1$, is commutative for every $x \in X$. The set $\Gamma\left(W, H_{n}\right)$ is a group with pointwise multiplication operation. Thus, the operation.: $H_{n} \oplus H_{n} \rightarrow H_{n}$ is continuous for every stalk of $H_{n}$ [1]. Hence, $H_{n}$ is a sheaf of abelian groups.

\section{CHARACTERISTIC Features OF $H_{n}$ [3]}

* Every section over an open set W can be extended to a section over $X$. In other words, the sections over $W$ are the restrictions of the sections over $X$, i.e., $\Gamma\left(W, H_{n}\right)=\Gamma\left(s \mid W, H_{n}\right), s \in \Gamma\left(X, H_{n}\right)$. A section over $\mathrm{X}$ is called a global section.

* All of the stalks of the sheaf $H_{n}$ over $X$ are isomorphic.

* Let $\mathrm{W} \subset \mathrm{X}$ be an open set and $\mathrm{s}_{1}, \mathrm{~s}_{2}$ be any two sections in $\Gamma\left(W, H_{n}\right)$. If $s_{1}\left(x_{0}\right)=s_{2}\left(x_{0}\right)$ for any $x_{0} \in W$, then $s_{1}(x)=s_{2}(x)$ for each $\mathrm{x} \in \mathrm{W}$.

* Let $\mathrm{W}_{1}, \mathrm{~W}_{2} \subset \mathrm{X}$ be any two open sets in $\mathrm{X}, \mathrm{W}_{1} \cap \mathrm{W}_{2} \neq \emptyset$ and $s_{1} \in \Gamma\left(W_{1}, H_{n}\right), s_{2} \in \Gamma\left(W_{2}, H_{n}\right)$. If $s_{1}\left(x_{0}\right)=s_{2}\left(x_{0}\right)$ for any $x_{0} \in W_{1}^{2} \cap W_{2}$, then $s_{1}(x)=s_{2}(x)$, for every $x \in W_{1} \cap W_{2}$. 


\section{THE SHEAF $H_{n}$ AS A COVERING SPACE}

Now, we shall prove that, $H_{n}$ is a regular covering space of $X$.

Theorem 3.1. Let $H_{n}$ be the sheaf of abelian groups over $(X, c)$ and $\mathrm{W}$ be an open set in $\mathrm{X}$. Then

$$
\left(\mathrm{H}_{\mathrm{n}}\right)_{\mathrm{c}} \cong \Gamma\left(\mathrm{W}, \mathrm{H}_{\mathrm{n}}\right) \text {. }
$$

Proof. Let $W \subset X$ be an open set and $s \in \Gamma\left(W, H_{n}\right)$. Then, there exists a unique element $\sigma_{c}=[\alpha]_{c} \subset\left(H_{n}\right)_{c}$ such that

$$
s(x)=\gamma^{*}\left([\alpha]_{c}\right)=[\beta]_{x}
$$

for every $x \in W$. That is, to each element of $\left(H_{n}\right)_{c}$, there correspondence only one element in $\Gamma\left(W, H_{n}\right)$. Let us denote this correspondence by $\Phi$ : $\left(H_{n}\right)_{c} \rightarrow \Gamma\left(W, H_{n}\right)$ such that $\Phi\left(\sigma_{c}\right)=s$ for any $\sigma_{c} \in\left(H_{n}\right)_{c}$. Let $\sigma_{c}{ }^{1}=$ $\left[\alpha_{1}\right]_{c}, \sigma_{c}{ }^{2}=\left[\alpha_{2}\right]_{c} \in\left(H_{n}\right)_{c}$ and $\sigma_{c}{ }^{1}, \sigma_{c}{ }^{2}$ determine the sections $s_{1}, s_{2} \in$ $\Gamma\left(\mathrm{W}, \mathrm{H}_{\mathrm{n}}\right)$, respectively. Then

$$
s_{1}(x)=\gamma^{*}\left(\left[\alpha_{1}\right]_{c}\right)=\left[\beta_{1}\right]_{x}
$$

and

$$
s_{2}(x)=\gamma^{*}\left(\left[\alpha_{2}\right]_{c}\right)=\left[\beta_{2}\right]_{x}
$$

for every $x \in W$. Then $s_{1}(x) \neq s_{2}(x)$, if $\sigma_{c}^{1} \neq \sigma_{c}^{2}$. So $\Phi$ is one to one. As a result of the definition of $\Phi, \Phi$ is onto. Thus $\Phi$ is a bijection.

$\Phi$ is a homomorphism. Because, if $\sigma_{c}{ }^{1}=\left[\alpha_{1}\right]_{c}, \sigma_{c}{ }^{2}=\left[\alpha_{2}\right]_{c} \in\left(H_{n}\right)_{c}$, then $\sigma_{c}{ }^{1} \cdot \sigma_{c}{ }^{2}=\left[\alpha_{1} \alpha_{2}\right]_{c}$. So the element $\sigma_{c}{ }_{c}^{1} \cdot \sigma_{c}{ }^{2} \in\left(H_{n}\right)_{c}$ defines a section $s \in \Gamma\left(\mathrm{W}, \mathrm{H}_{\mathrm{n}}\right)$ such that

$$
s(x)=\left(s_{1} \cdot s_{2}\right)(x)=\gamma^{*}\left(\left[\alpha_{1} \cdot \alpha_{2}\right]_{c}\right)=\left[\beta_{1} \cdot \beta_{2}\right]_{x}
$$

for every $x \in W$. On the other hand for every $x \in W$,

$$
\begin{aligned}
s_{1}(x) \cdot s_{2}(x) & =\gamma^{*}\left(\left[\alpha_{1}\right]_{c}\right) \cdot \gamma^{*}\left(\left[\alpha_{2}\right]_{c}\right) \\
& =\gamma^{*}\left(\left[\alpha_{1}\right]_{c}\left[\alpha_{2}\right]_{c}\right) \\
& =\gamma^{*}\left(\left[\alpha_{1} \cdot \alpha_{2}\right]_{c}\right) \\
& =\left[\beta_{1} \cdot \beta_{2}\right]_{x} .
\end{aligned}
$$


Thus

$$
\Phi\left(\sigma_{c}^{1} \cdot \sigma_{c}^{2}\right)=s_{1} s_{2}=\Phi\left(\sigma_{c}{ }^{1}\right) \cdot \Phi\left(\sigma_{c}^{2}\right)
$$

Therefore, $\Phi$ is an isomorphism.

We can state as a results of Theorem 3.1. that, the stalk $\left(\mathrm{H}_{\mathrm{n}}\right)_{\mathrm{c}}$ completely determines the group of sections over W. In particular, if we take $W=X$, then the stalk $\left(H_{n}\right)_{c}$ completely determines the group of global sections over $\mathrm{X}$.

Now we can state the following corollary [2].

Corollary. Let $H_{n}$ be the sheaf of abelian groups over $X,\left(H_{n}\right)$ be the stalk over the point $x \in X$ and $W=W(x)$ be an open set. Then, $\left(H_{n}\right)_{x} \cong \Gamma\left(W, H_{n}\right)$. Particularly, $\left(H_{n x} \cong \Gamma\left(X, H_{n}\right)\right.$.

According to this corollary, we can say that, if $\sigma_{x} \in\left(H_{n x}\right)$ is any element and $W=W(x)$ is an open set in $X$, then there is a unique section $s \in \Gamma\left(W, H_{n}\right)$ such that $s(x)=\sigma_{x}$. Since

$$
\Psi \mid s(W): s(W) \rightarrow W
$$

is a topological mapping and $s=(\Psi \mid s(W))^{-1}$,

$$
\Psi^{-1}(W)=\underset{i \in I}{V_{i}} s_{i}(W), s_{i} \in \Gamma\left(W, H_{n}\right)
$$

and

$$
\Psi \mid \mathbf{s}_{i}(W): s_{i}(W) \rightarrow W
$$

is a topological mapping. So, the open set $\mathrm{W}=\mathrm{W}(\mathrm{x})$ is evenly covered by $\Psi$. Thus $\Psi$ is a covering projection and $\left(H_{n}, \Psi\right)$ is a covering space of $X[7,8,9]$. Moreover, $\left(H_{n}, \Psi\right)$ is an abelian covering space of $X$.

Now, let $x_{0} \in X$ be any point and $\gamma$ be an arc with initial point $x_{0}$, Then, the mapping

$$
\text { soy : } \mathrm{I} \rightarrow \mathrm{H}_{\mathrm{n}}
$$

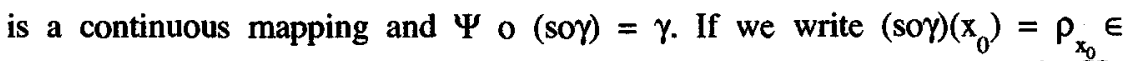
$\left(H_{n}\right)_{x_{0}}$, then sor is a lifting of $\gamma$ from the initial point $\rho_{x_{0}}$ over $x_{0}$ in $H_{n}$. 
Write so $\gamma=\gamma^{*}$, then $\gamma^{*}$ is unique, because the mapping $\Psi \mid s(X)$ : $\mathrm{s}(\mathrm{X}) \rightarrow \mathrm{X}$ is a homeomorphism.

We can then state the following theorem.

Theorem 3.2. Let $\left(H_{n}, \Psi\right)$ be the sheaf of abelian groups over $X, x_{0}$ $\epsilon \mathrm{X}$ be any point and $\gamma^{n}$ be a path with initial point $x_{0}$ in $X$. Then, $\gamma$ has a unique lifting $\gamma^{*}$ with initial point $\rho_{x_{0}}$ in $H_{n}$, for $\rho_{x_{0}} \in\left(H_{n}\right)_{x_{0}}$.

Now, we give the following theorem.

Theorem 3.3. (Monodromy). Let $\left(H_{n}, \Psi\right)$ be the sheaf of abelian groups over $\mathrm{X}$ and suppose that $\gamma_{1}^{*}$ and $\gamma_{2}^{*}$ are paths with common initial point $\rho_{x_{0}}$ and terminal point $\rho_{x_{1}}$ in $\hat{H}_{n}$. Then, $\gamma_{1}{ }^{*}$ and $\gamma_{2}^{*}$ are homotopic path in $\mathrm{H}_{\mathrm{n}}$ if and only if $\Psi o \gamma_{1}^{*}$ and $\Psi o \gamma_{2}^{*}$ are homotopic paths in $X$.

Proof. If $\gamma_{1}^{*}$ is homotopic to $\gamma_{2}^{*}$ by a homotopy $\mathrm{G}$, then $\Psi \circ G$ is a homotopy between $\Psi o \gamma_{1}{ }^{*}$ and $\Psi o \gamma_{2}{ }^{*}$. For a proof of the other half of the theorem, let $x_{0}$ and $x_{1}$ denote the common initial point and common terminal point $\Psi \mathrm{o} \gamma_{1}{ }^{*}$ and $\Psi \mathrm{o} \gamma_{2}{ }^{*}$, respectively. Let $\mathrm{H}: \mathrm{I} \mathrm{x} \mathrm{J} \rightarrow \mathrm{X}$ be a homotopy between $\Psi o \gamma_{1}^{*}$ and $\Psi o \gamma_{2}{ }^{*}$. On the other hand, if $\rho_{x_{0}} \in\left(H_{n}\right)$, then there is a unique section $s \in \Gamma\left(X, H_{n}\right)$ such that $s\left(x_{0}\right)=\rho_{x_{0}}$. So,

$$
\operatorname{so}\left(\Psi o \gamma_{1}^{*}\right)=\gamma_{1}^{*}
$$

and

$$
\operatorname{so}\left(\Psi o \gamma_{2}^{*}\right)=\gamma_{2}^{*}
$$

Furthermore, soH is a homotopy between $\gamma_{1}^{*}$ and $\gamma_{2}^{*}$.

Theorem 3.4. Let $\left(H_{n}, \Psi\right)$ be the sheaf of abelian groups over $X, x_{0}$ $\in X$ be an arbitrary fixed point and $\rho_{x_{0}} \in\left(H_{n}\right)_{x_{0}}$ be any point. Then the fundamental group of $H_{n}$ with respect to $\rho_{x_{0}}$ is isomorphic to $\left(H_{n}\right)_{x_{0}}$. $\mathrm{X}$.

From theorems 3.3. and $3.4,\left(H_{n}, \Psi\right)$ is a regular covering space of

Now, we give "General Lifting Theorem" for the sheaf $\mathrm{H}_{\mathrm{n}}$.

Theorem 3.5. Let $X=\left(X, x_{0}\right), Y=\left(Y, y_{0}\right)$ be two connected and locally path connected topological space (or two Riemann spaces), $\left(\mathrm{H}_{n}, \Psi\right.$ ) 
be the sheaf of abelian groups over the pointed topological space $\left(X, x_{0}\right)$, $\rho_{x_{0}} \in \Psi^{-1}\left(x_{0}\right)$ be any point. If

$$
f:\left(Y, y_{0}\right) \rightarrow\left(X, x_{0}\right)
$$

be any continuous mapping, then $f$ can be lifted to a unique continuous

$$
\mathrm{f}^{*}:\left(\mathrm{Y}, \mathrm{y}_{0}\right) \rightarrow\left(\mathrm{H}_{\mathrm{n}}, \rho_{\mathrm{x}_{0}}\right)
$$

such that $\Psi$ of $=\mathrm{f}^{*}$.

Proof. Let $\mathrm{f}:\left(\mathrm{Y}, \mathrm{y}_{0}\right) \rightarrow\left(\mathrm{X}, \mathrm{x}_{0}\right)$ be a continuous mapping. Then $f\left(y_{0}\right)=x_{0}$. If $\rho_{x_{0}} \in \Psi^{-1}\left(x_{0}\right)$ any point, then there exists a unique section $s \in \Gamma\left(X, \stackrel{H}{n}_{n}\right)$ such that $s\left(x_{0}\right)=\rho_{x_{0}}$. Thus

$$
\text { sof }:\left(Y, y_{0}\right) \rightarrow\left(H_{n}, \rho_{x_{0}}\right)
$$

is a continuous mapping and

$$
\Psi \text { o(sof) }=\text { f }
$$

So, sof is a lifting of $f$ to $H_{n}$. Let us denote sof by $f^{*}$. $f^{*}$ is unique, because the section $s$ is unique.

We can now state the following theorem.

Theorem 3.6. Let $\mathrm{X}=\left(\mathrm{X}, \mathrm{x}_{0}\right), \mathrm{Y}=\left(\mathrm{Y}, \mathrm{y}_{0}\right)$ be two connected and locally path connected topological space (or two Riemann Surfaces), $\left(\mathrm{H}_{\mathrm{n}}\right.$, $\Psi$ ) be the sheaf of abelian groups over the pointed topological space (X, $\left.\mathrm{x}_{0}\right), \rho_{\mathrm{x}_{0}} \in \Psi^{-1}\left(\mathrm{x}_{0}\right)$ be any point and

$$
f^{*}, g^{*}:\left(Y, y_{0}\right) \rightarrow\left(H_{n}, \rho_{x_{0}}\right)
$$

be any two continuous mappings such that $\Psi_{0}^{*}=\Psi o g^{*}$, then

$$
\mathbf{f}^{*}=\mathrm{g}^{*} \text {. }
$$

Proof. This is a result of Theorem 3.5 .

\section{SUBSHEAVES AND QUOTIENT SHEAVES OF $H_{n}$.}

In this section, Constructing the Quotient sheaf $Q_{H_{n}^{\prime}}$, for any subsheaf of the groups $\mathrm{H}_{n}^{\prime}$ of the sheaf $\mathrm{H}_{\mathrm{n}}$, it is shown that $\mathrm{Q}_{\mathrm{H}_{\mathrm{n}}^{\prime}}$ is covering space as a sheaf of abelian groups. 
We begin by giving the following definition [5].

Definition 4.1. Let $H_{n}$ be the sheaf of abelian groups over $X$ and $\mathrm{H}^{\prime} \subset \mathrm{H}_{\mathrm{n}}$ be an open set. Then $\mathrm{H}_{\mathrm{n}}^{\prime}$ is called a subsheaf of the sheaf $\mathrm{H}_{\mathrm{n}}$ of abelian groups, if

i) $\Psi\left(\mathrm{H}_{\mathrm{n}}^{\prime}\right)=X$

ii) For each point $x \in X$, the stalk $\left(H_{n}^{\prime}\right)_{x}$ is a subgroup of $\left(H_{n}\right)_{x}$.

We now give the following theorem.

Theorem 4.1. (Existence Theorem). Let $X=(X, c)$ be a connected, locally path connected topological space and $\left(\mathrm{H}_{\mathrm{n}}\right)_{\mathrm{c}}$ be higher homotopy group with respect to $c \in X$. Then each subset $\left(\mathrm{H}_{n^{\prime}}\right)_{c}$ of $\left(\mathrm{H}_{n}\right)_{c}$ determines a sheaf over $X$.

As a result of Theorem 4.1.,

1. If $\left(\mathrm{H}_{\mathrm{n}_{\mathrm{c}}^{\prime}}\right)_{\mathrm{n}}=\left(\mathrm{H}_{\mathrm{n}}\right)_{\mathrm{c}}$, it is obtained that $\mathrm{H}_{\mathrm{n}}^{\prime}=\mathrm{H}_{\mathrm{n}}$. So, the sheaves $\mathrm{H}_{\mathrm{n}}^{\prime}$ are subsheaves of $\mathrm{H}_{\mathrm{n}}$. Also, $\Psi^{\prime}=\Psi \mathrm{H}_{\mathrm{n}}^{\prime}$. $\left(\mathrm{H}_{\mathrm{n}_{2}}^{\prime}\right)_{\mathrm{c}}$ then

2. If $\left(\mathrm{H}_{n_{1}^{\prime}}^{\prime}\right)_{c},\left(\mathrm{H}_{n_{2}}\right)_{c}$ are any two subset of $\left(\mathrm{H}_{\mathrm{n}_{\mathrm{c}}}^{\prime}\right)_{\mathrm{c}}$ and $\left(\mathrm{H}_{\mathrm{n}_{1}}^{\prime}\right)_{\mathrm{c}} \subset$ $\mathrm{H}_{\mathrm{n}_{1}}^{\prime} \subset \mathrm{H}_{\mathrm{n}_{2}}^{\prime}$.

Furthermore, if $\mathrm{W} \subset \mathrm{X}$ is an open set, then

$\Gamma\left(\mathrm{W}, \mathrm{H}_{\mathrm{n}_{1}^{\prime}}^{\prime}\right) \subset \Gamma\left(\mathrm{W}, \mathrm{H}_{\mathrm{n}_{2}}^{\prime}\right) \subset \Gamma\left(\mathrm{W}, \mathrm{H}_{\mathrm{n}}\right)$.

3. Let $\mathrm{H}_{\mathrm{n}}^{\prime}$ be a subsheaf of the sheaf $\mathrm{H}_{n}$ of abelian groups and $\mathrm{W}$ $\subset X$ be an open set. Then $\Gamma\left(W, H_{n}^{\prime}\right)$ is a subgroup of $\Gamma\left(W, H_{n}\right)$. If we take $W=X$, then $\Gamma\left(X, H_{n}^{\prime}\right)$ is a subgroup of $\Gamma\left(X, H_{n}\right)$.

Now, we give the following definition.

Definition 4.2. Let $H_{n}$ be the sheaf of abelian groups over $X$ and $\mathrm{H}_{\mathrm{n}}^{\prime} \subset \mathrm{H}_{\mathrm{n}}$ be a subsheaf of abelian groups. Let us associate the set

$$
\mathrm{M}_{\mathrm{W}}=\Gamma\left(\mathrm{W}, \mathrm{H}_{\mathrm{n}}\right) / \Gamma\left(\mathrm{W}, \mathrm{H}_{\mathbf{n}}^{\prime}\right)
$$


with the open set $W$, for each $W \subset X$ open. Then, the system $\left\{X, M_{W}\right.$, $\left.\gamma_{\mathrm{w}, \mathrm{V}}\right\}$ is a pre-sheaf [4]. The sheaf defined by the pre-sheaf $\left\{X, M_{w}\right.$, $\left.\gamma_{\mathrm{w}, \mathrm{V}}\right\}$ is called Quotient sheaf and it is denoted by $\mathrm{Q}_{\mathrm{H}_{\mathrm{n}}}$.

Theorem 42. Let $H_{n}$ be the sheaf of abelian groups over $X$ and $H_{n}^{\prime}$ $\subset H_{n}$ be a subsheaf of abelian groups. Then, the Quotient sheaf $Q_{H_{n}^{\prime}}$ is a sheaf of abelian groups over $\mathrm{X}$.

Proof. Let $H_{n}$ be the sheaf of abelian groups over $X$ and $H_{n}^{\prime} \subset H_{n}$ be a subsheaf of abelian groups. Also, $H^{\prime}$ is a normal subsheaf of the sheaf $H_{n}$. So, $\Gamma\left(X, H_{n}^{\prime}\right) \subset \Gamma\left(X, H_{n}\right)$ is a normal subgroup and $\Gamma\left(X, H_{n}\right) /\left(X, H_{n}^{\prime}\right)$ is a group. Let

$$
\mathrm{Q}_{\mathrm{H}_{\mathrm{n}}^{\prime}}=\underset{\mathrm{x} \in \mathrm{X}}{\bigvee}\left(\mathrm{Q}_{\mathrm{H}_{\mathrm{n}}{ }_{\mathrm{x}}}\right)
$$

and

$$
\left(Q_{H_{n}^{\prime}}\right)_{x}=\left\{(W,[s])_{x}: W \subset X \text { is an open set, }[s] \in \Gamma\left(X, H_{n}\right) / \Gamma\left(X, H_{n}^{\prime}\right)\right\} \text {. }
$$

So, the operation defined in each stalk $\left(\mathrm{Q}_{\mathrm{H}_{\mathrm{n}}^{\prime}}\right)_{\mathrm{x}}$ in the form of $\left(\mathrm{W},\left[\mathrm{s}_{1}\right]\right)_{\mathrm{x}}$. $\left(W,\left[s_{2}\right]\right)_{x}=\left(W,\left[s_{1}, s_{2}\right]\right)_{x}$ is well defined. It is easily seen that each stalk $\left(Q_{H_{n}^{\prime}}\right)_{x}$ is an abelian group with this operation for every $x \in X$. Since $\left(\mathrm{Q}_{\mathrm{H}_{\mathrm{n}}^{\prime}}\right)_{\mathrm{x}} \cong \Gamma\left(\mathrm{X}, \mathrm{Q}_{\mathrm{H}_{\mathrm{n}}^{\prime}}\right), \Gamma\left(\mathrm{X}, \mathrm{Q}_{\mathrm{H}_{\mathrm{n}}^{\prime}}\right)$ is an abelian group. Thus, $\mathrm{Q}_{\mathrm{H}_{\mathrm{n}}^{\prime}}$ is a sheaf of abelian groups.

Moreover, $\mathrm{Q}_{\mathrm{H}_{\mathrm{n}}^{\prime}}$ is a covering space as a sheaf of abelian groups. Also, it is a regular covering space.

Theorem 4.3. Let $H_{n}$ be the sheaf of abelian groups over $X, H_{n}^{\prime} \subset$ $H_{n}$ be a subsheaf of abelian groups and $\mathrm{Q}_{\mathrm{H}^{\prime}}$ be quotient sheaf. Then the group $\Gamma\left(\mathrm{X}, \mathrm{Q}_{\mathrm{H}_{\mathrm{n}}}\right)$ is isomorphic to the quotient group $\Gamma\left(\mathrm{X}, \mathrm{H}_{\mathrm{n}}\right) / \Gamma\left(\mathrm{X}, \mathrm{H}_{\mathrm{n}}^{\prime}\right)$.

Proof. To prove this theorem let us define the mapping

$$
\gamma: \Gamma\left(\mathrm{X}, \mathrm{H}_{\mathrm{n}}\right) / \Gamma\left(\mathrm{X}, \mathrm{H}_{\mathrm{n}}^{\prime}\right) \rightarrow \Gamma\left(\mathrm{X}, \mathrm{Q}_{\mathrm{H}_{\mathrm{n}}^{\prime}}\right)
$$

in the form of $\gamma([\mathrm{s}])=\gamma[\mathrm{s}]$, where $\gamma$ representes inductive limit [4]. If $\gamma([\mathrm{s}])$ $=1$, then $\gamma[s]=1$ and so, $\gamma[s](x)=(X,[e])$, for any $x \in X$. That is

$$
(\mathrm{W},[\mathrm{s}])_{\mathrm{x}}=(\mathrm{W},[\mathrm{e}])_{\mathrm{x}} \text {. }
$$


Thus,

$$
[\mathbf{s}]=[\mathrm{e}] \text {. }
$$

Hence, $\gamma$ is one to one. Clearly $\gamma$ is onto. Now, if $\left[s_{1}\right],\left[s_{2}\right] \in \Gamma\left(X, H_{n}\right)$ $/ \Gamma\left(\mathrm{X}, \mathrm{H}_{\mathrm{n}}^{\prime}\right)$ are any two elements, then

$$
\begin{aligned}
\gamma\left(\left[\mathrm{s}_{1}\right]\left[\mathrm{s}_{2}\right]\right) & =\gamma\left(\left[\mathrm{s}_{1} \cdot \mathrm{s}_{2}\right]\right) \\
& =\gamma\left[\mathrm{s}_{1} \mathrm{~s}_{2}\right] \\
& =\gamma\left[\mathrm{s}_{1}\right] \cdot \gamma\left[\mathrm{s}_{2}\right]
\end{aligned}
$$

Thus, $\gamma$ is a homomorphism.

Therefore, $\gamma: \Gamma\left(\mathrm{X}, \mathrm{H}_{\mathrm{n}}\right) / \Gamma\left(\mathrm{X}, \mathrm{H}_{\mathrm{n}}^{\prime}\right) \rightarrow \Gamma\left(\mathrm{X}, \mathrm{Q}_{\mathrm{H}_{\mathrm{n}}^{\prime}}\right)$ is an isomorphism.

\section{REFERENCES}

[1] BALCI, S., On the Restricted Sheaf, Comm. Fac. Sci. Univ. Ankara Ser. A1, Vol 37 (1988) pp. 41-51.

[ 2] BALCI, S., On the Existence and Lifting Theorems for Sheaves, Pure and Applied Mathematika Sciences, Vol. 37, No. 1-2 (1993) pp., 57-65.

[ 3] BALCl, S., The Seifert-Van Kampen Theorem for the Group of Global Sections, Indian J. Pure and Appl. Math., 27(9) (1996) pp., 883-891.

[ 4] GRAURET, H. and FRITZSCHE, K., Several Complex Variables, Springer-Verlag, New York (1976).

[ 5] GUNNING, R.C. and ROSSI, H., Analytic Function of Several Variables, Prentice-Hall, Inc., Englewood Cliffs, N.T. (1965).

[ 6] HILTON, P.J., An Introduction to Homotopy Theory, Cambridge University Press., Cambridge (1961).

[ 7] MUNKERS, J.R., Topology, Prentice Hall, Inc., Englewood Cliffs, New Jersey (1975).

[ 8] ULUÇAY, C., Restricted Sheaf Theory, Journal of Fac. Sci. of the K.T.Ü., Vol III, Fasc.7 (1980) pp., 59-68.

[ 9] YILDIZ, C., The Sheaf of the Homology Groups of the Complex Manifolds, Comm. Fac. Sci. Univ. Ankara, Ser A1 Mathematiques, Tom: 46 (1997).

[10] YILDIZ, C., The Sheaf of the H-Groups, Journal of the Institute of Sci. and Technology of Gazi Univ., Vol.10 No:2, (1997). 